\title{
Renal Papillary Necrosis
}

Farid S. Haddad, M.D.

Phoenix, Arizona

DOI: http://dx.doi.org/10.5915/23-2-15085

\begin{abstract}
The symptoms of renal papillary necrosis may mimick those of ureteral lithiasis. A case report is presented with a discussion on etiology, clinical course, reontgenology, and pathology of the condition.
\end{abstract}

Key words: Papillary necrosis, kidney, analgesic abuse

Renal papillary necrosis (RPN), also called necrotizing papillitis or renal medullary necrosis, is characterised by necrosis of the renal papilla followed by sloughing. The sloughed tissue may get extruded via the ureter, get impacted in the ureter or may remain in the kidney and become calcified. In about $12 \%$ of the patients the disease has a fulminating picture and in half of the cases, a nephrectomy may be necessary ${ }_{z}{ }^{2}$

\section{Case report}

A 62-year-old white male presented with acute sharp pain in the right flank. He had been in chronic renal failure with a creatinine that had stabilised at about $6.5 \mathrm{mg} \%$. Presumably, the renal failure was the result of prolonged use of a phenacetin containing medication.

The right kidney was palpable and tender. The blood pressure was $140 / 90$. Urinalysis revealed a

From the Urology Section

Carl T. Hayden Veterans Administration

Medical Center

Phoenix, Arizona

Reprint Requests: Farid S. Haddad, M.D.

4332 E. Piccadilly Road

Phoenix, AZ 85018 specific gravity of 1.010 , a pH of 6.0 , one plus protein and 5 red blood cells per high power field. The blood urea nitrogen was $84 \mathrm{mgs} \%$, the creatinine 9 $\mathrm{mg} \%$, the potassium $4.5 \mathrm{mg} \%$ and the hematocrit 24.

The clinical impression was that of right hydronephrosis. A retrograde pyelogram revealed ureteral obstruction at $22 \mathrm{~cm}$ from the ureteral orifice (Figure 1). A right percutaneous nephrostomy was then performed. The antegrade pyelogram revealed a filling defect in the right lower ureter (Figure 2); the obstruction had moved down the ureter.

After climbing to $11 \mathrm{mg} \%$, the creatinine began to go down again. Six days later, the patient passed a $0.8 \times 0.3 \mathrm{~cm}$ "brown clot" (Figure 3 ). It was sent for microscopic examination and found to be a sloughed necrotic renal papilla (Figure 4).

The patient developed a post obstructive diuresis. His blood returned to normal $(115 / 70)$ and he was discharged a week after passing the sloughed renal papilla.

\section{Discussion}

It was originally thought that RPN was associated only with diabetes mellitus; in fact the incidence of RPN in IDDM is of the order of $24 \% .^{3}$ At present, RPN is known to be associated with several other conditions (see table). ${ }^{1}$

Colicky pain, high fever as well as pyuria and hematuria are frequently but not invariably found. Septicemia has been described. The clinical course 


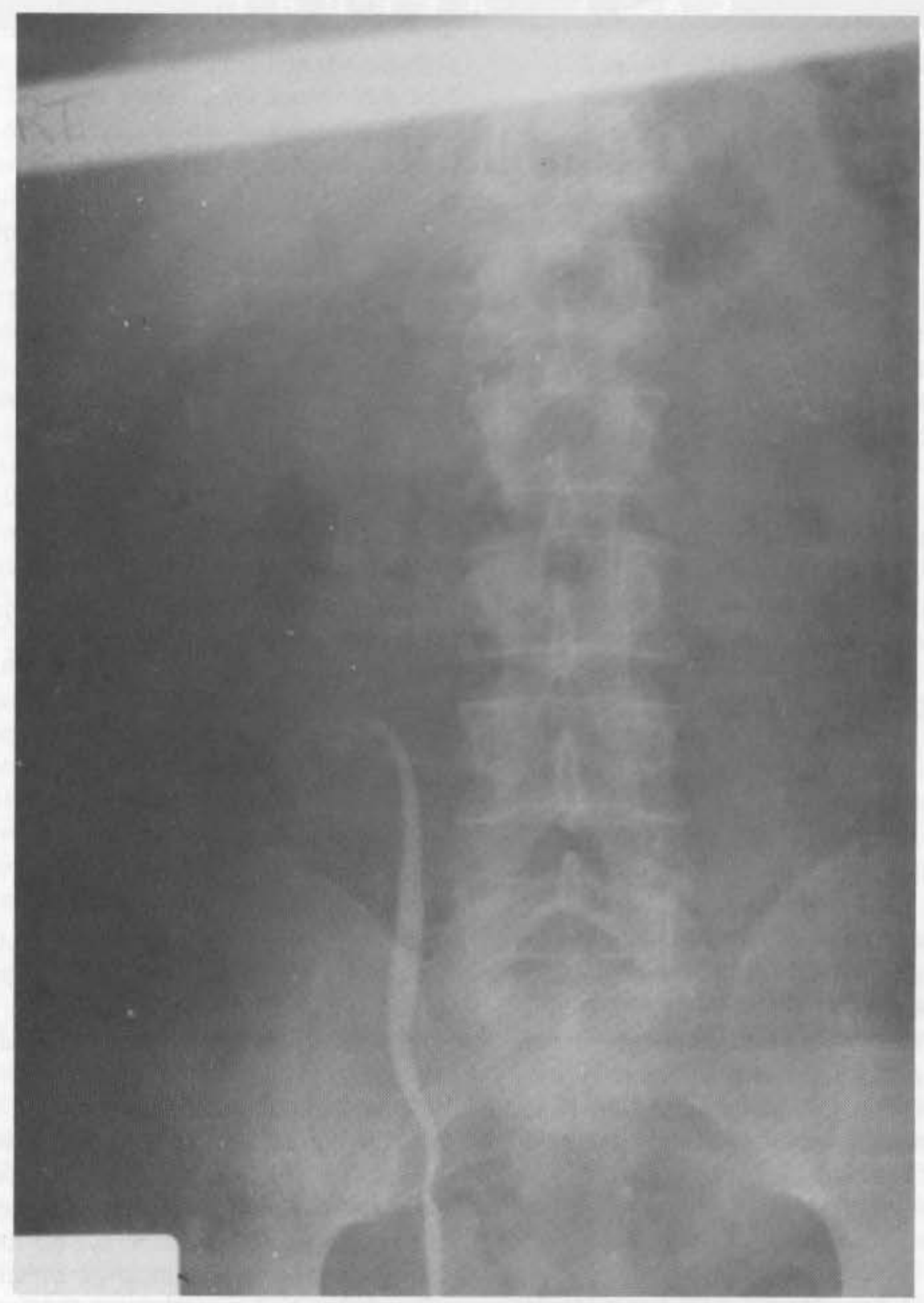

Figure 1 Retrograde pyelogram showing obstruction of the right ureter.

Table 1. - Conditions associated with renal papillary necrosis.
Diabetes
Phenacetin (prolonged use)
Sickle cell disease
Ureteral obstruction with hydronephrosis
Pyelonephritis
Renal vein thrombosis
Severe dehydration
Transplant rejection 


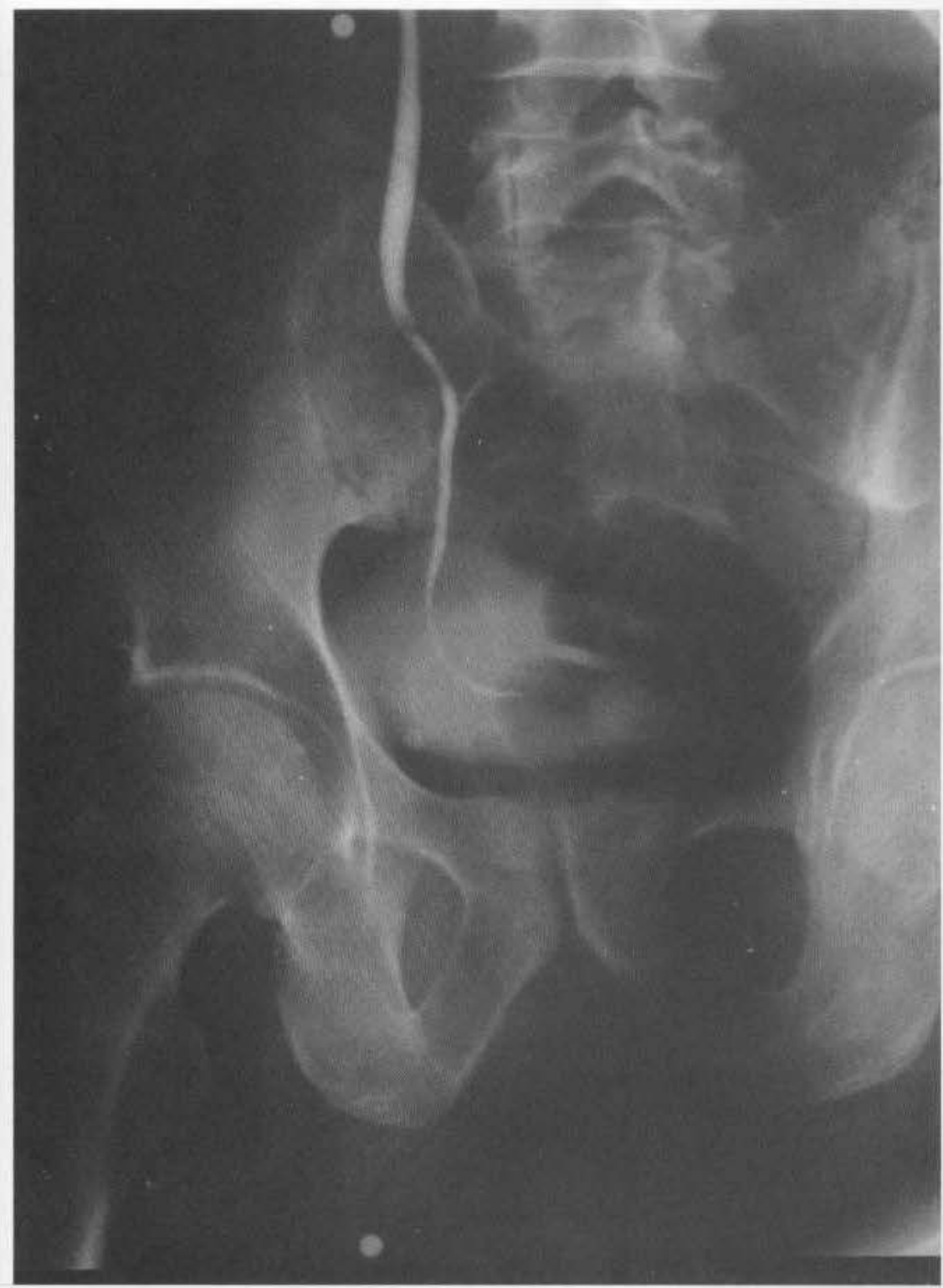

Figure 2

Antegrade pyelo-ureterogram showing a ureteral filling defect.

Figure 3

Sloughed necrotic renal papillary tissue, gross specimen recovered from the urine. 
Figure 4 Necrotic renal papilla, microscopic view.

may be mild, even asymptomatic, or the sloughed papilla can cause prolonged ureteral obstruction, severe renal damage and sometimes catastrophic loss of renal tissue and death. ${ }^{1}$

The roentgenologic findings include: a typical ring sign, blurring of the papillary outline, cavitation in the pyramid or medulla, and a ureteral filling defect. ${ }^{1}$

The lesion of RPN was first described by Friedreich in 1877. Grossly, it involves one or more pyramids, unilaterally or bilaterally; the pyramid may be partially or completely replaced by whitish or yellowish grey necrotic foci. Microscopically there is ischemic infarction and coagulative necrosis of the tip of the pyramid including both interstitial tissue and tubular epithelium. A purulent exudate may be seen within and between the collecting tubules. In some cases this necrosis and this exudate may extend to various distances up the medulla where small abscess cavities may be seen along with medullarypelvic sinus tracts. ${ }^{3}$ It has been recently proposed that the post-obstructive diuresis could be due to an observed three fold increase in atrial natriuretic peptide. ${ }^{4}$

\section{References}

1. Haddad Farid S: Renal papillary necrosis. In: Kovac A: Guide to diagnostic imaging. Vol 4, (ed) Haddad FS: Upper urinary tract and adrenal gland. New York: Med Exam Publ. Co Inc, Med. Excerpta Co., 1984 chap 8:199-200.

2. Flaster S, Lome LG et al: Urologic complications of renal papillary necrosis. Urology 1975; $5(3): 331-6$.

3. Groop L, Laasonen L, et al: Renal papillary necrosis in patients with IDDM. Diabetes Care 1989; 12(3):192-202.

4. Gulmi FA, Mooppan UMM, et al: Atrial natriuretic peptide in patients with obstructive uropathy. J Urol 1989; 142(2):268-72. 